\title{
MULTI-PARAMETER BURAU REPRESENTATIONS
}

\author{
MADLINE AL-TAHAN, MOHAMMAD N. ABDULRAHIM AND SAMER S. HABRE
}

\begin{abstract}
We consider the multi-parameter representation of Artin's braid group introduced by D. D. Long and J. P. Tian, namely $\alpha: B_{n} \rightarrow G L_{m}(C)$, where $m=n ! n$. First, we show that there exists a complex specialization of the multi-parameter representation that does not arise from any Hecke algebra. Second, we find conditions under which the images of the generators of the braid group on three strings under the multi-parameter representation are unitary relative to a nonsingular hermitian matrix.
\end{abstract}

\section{Introduction}

The braid group, $B_{n}$, has a well- known representation due to $\operatorname{Artin}$ in the group $\operatorname{Aut}\left(F_{n}\right)$ of automorphisms of the free group $F_{n}$ generated by $x_{1}, \ldots, x_{n}$. The automorphism corresponding to the braid generator $\sigma_{i}$ takes $x_{i}$ to $x_{i} x_{i+1} x_{i}^{-1} ; x_{i+1}$ to $x_{i}$, and fixes all other free generators. Applying the Fox derivatives and Magnus representation, we get the classical Burau representation which is of degree $n$. In [3], D.D. Long gave a new derivative of the Burau representation by using an action of $\operatorname{Aut}\left(F_{n}\right)$ on the representation variety $R=R\left(F_{n}, G\right)$, where $G$ is a compact semisimple Lie group and $F_{n}$ is the free group of rank $n$.

In Section 2, we define the multi-parameter representation, namely $\alpha: B_{n} \rightarrow G L_{m}(\mathbb{C})$, where $m=n ! n$. Explicit matrices will be computed in the case $n=3$. For more details, see [6]. In Section 3, we prove that there exist complex specializations of the multi-parameter representation that do not arise from any Hecke algebra. In Section 4, we determine sufficient conditions under which the complex specializations of the multi-parameter representation of the braid group on three strings is unitary relative to a nonsingular hermitian matrix .

\section{Definitions}

Definition 1 ([2]). The braid group on $n$ strings, $B_{n}$, is the abstract group with presentation $B_{n}=\left\{\sigma_{1}, \ldots, \sigma_{n-1} / \sigma_{i} \sigma_{i+1} \sigma_{i}=\sigma_{i+1} \sigma_{i} \sigma_{i+1}\right.$ for $i=1,2, \ldots, n-2, \sigma_{i} \sigma_{j}=\sigma_{j} \sigma_{i}$ if $\left.|i-j|>1\right\}$. The generators $\sigma_{1}, \ldots, \sigma_{n-1}$ are called the standard generators of $B_{n}$.

Corresponding author: Mohammad N. Abdulrahim. 2010 Mathematics Subject Classification. Primary 20F36.

Key words and phrases. Braid group, Burau representation, unitary. 
Definition 2. [2] The pure braid group, denoted by $P_{n}$, is defined as the kernel of the homomorphism $B_{n} \rightarrow S_{n}$ defined by $\sigma_{i} \rightarrow(i, i+1), 1 \leq i \leq n-1$. It is finitely generated by the elements

$$
A_{i j}=\sigma_{j-1} \cdots \sigma_{i+1} \sigma_{i}^{2} \sigma_{i+1}^{-1} \cdots \sigma_{j-1}^{-1}, 1 \leq i<j \leq n .
$$

Definition 3. Let $G$ be a finitely generated group. A representation $G \rightarrow G L_{r}(C)$ is reducible if there exists a non-zero proper subspace of $C^{r}$ that is invariant under the action of the generators of $G$.

Applying the new derivative of the Burau representation adopted by D. D. Long in [3], J.P. Tian has made some computations by considering the action of the braid group $B_{n}$ on a special case of a representation variety, namely $R=R\left(F_{n}, G\right)$, where $G=S U(2, \mathbb{C})$. This gives us the definition of the multi-parameter representation.

Let us consider $V_{t}$ as a linear space which is a summation of all tangent spaces of $R$ at its base points. $\beta \in \operatorname{Aut}\left(F_{n}\right)$ acts on $R$ and $\beta^{*}\left(p_{\theta}^{t}\right)=p_{\theta}^{\tau_{\beta}(t)}$, where $\tau_{\beta}$ is a permutation determined by $\beta$ and acting on $t$ in an obvious way.

Definition 4. [6] The multi-parameter representation of the braid group is a map $\alpha: B_{n} \rightarrow$ $G L\left(V_{t}\right)$, where $\alpha(\beta)=\oplus_{\tau \in S_{n}} d \beta_{p_{\theta^{\tau}(t)}^{*}}$. Here $\tau(t)$ is a permutation of $\left(t_{1}, t_{2}, \cdots, t_{n}\right) \in \mathbb{R}^{n}, \beta^{*} \in$ $\operatorname{Dif} f(R)$ and $p_{\theta^{\tau(t)}}$ are the base points.

For a particular permutation $t=\left(t_{1}, t_{2}, \cdots, t_{n}\right)$, we compute the matrix representations of the images of the generators of $B_{n}$ under the multi-parameter representation, namely $\alpha\left(\sigma_{k}\right)$. The order we give for the permutations of $\left(t_{1}, t_{2}, \cdots, t_{n}\right)$ is the lexicographic order.

For a fixed choice $t=(\ldots, \underbrace{t_{i}}_{k}, t_{j}, \ldots)$, we have that

$$
d\left(\sigma_{k}^{*}\right)_{p_{\theta}^{t}}=M_{k}\left(t_{i}, t_{j}\right)=I_{k-1} \oplus\left(\begin{array}{cc}
1-\lambda^{t_{j}} & \lambda^{t_{i}} \\
1 & 0
\end{array}\right) \oplus I_{n-k-1} .
$$

Here $k=1, \cdots, n-1$ and $1 \leq i \neq j \leq n$. We then compute $d\left(\sigma_{k}^{*}\right)_{p_{\theta}^{\tau(t)}}$, where $\tau$ is a permutation in $S_{n}$. We expect to have $n$ ! square matrices $M_{k}\left(t_{i}, t_{j}\right)$, each of size $n \times n$. In order to place these square $n \times n$ matrices $M_{k}\left(t_{i}, t_{j}\right)$ in the matrix $\alpha\left(\sigma_{k}\right)$, we simply order the $n$-tuples in the lexicographic order. In the case $n=3$, the tuples are ordered as follows: $\left(t_{1}, t_{2}, t_{3}\right),\left(t_{1}, t_{3}, t_{2}\right)$, $\left(t_{2}, t_{1}, t_{3}\right),\left(t_{2}, t_{3}, t_{1}\right),\left(t_{3}, t_{1}, t_{2}\right)$ and $\left(t_{3}, t_{2}, t_{1}\right)$. More precisely, the images of the generators of $B_{3}$ under the multi-parameter representation $\alpha: B_{3} \rightarrow G L_{18}(\mathbb{C})$ are given by

$$
\alpha\left(\sigma_{1}\right)=\left(\begin{array}{cccccc}
0 & 0 & M_{1}\left(t_{2}, t_{1}\right) & 0 & 0 & 0 \\
0 & 0 & 0 & 0 & M_{1}\left(t_{3}, t_{1}\right) & 0 \\
M_{1}\left(t_{1}, t_{2}\right) & 0 & 0 & 0 & 0 & 0 \\
0 & 0 & 0 & 0 & 0 & M_{1}\left(t_{3}, t_{2}\right) \\
0 & M_{1}\left(t_{1}, t_{3}\right) & 0 & 0 & 0 & 0 \\
0 & 0 & 0 & M_{1}\left(t_{2}, t_{3}\right) & 0 & 0
\end{array}\right)
$$


and

$$
\alpha\left(\sigma_{2}\right)=\left(\begin{array}{cccccc}
0 & M_{2}\left(t_{3}, t_{2}\right) & 0 & 0 & 0 & 0 \\
M_{2}\left(t_{2}, t_{3}\right) & 0 & 0 & 0 & 0 & 0 \\
0 & 0 & 0 & M_{2}\left(t_{3}, t_{1}\right) & 0 & 0 \\
0 & 0 & M_{2}\left(t_{1}, t_{3}\right) & 0 & 0 & 0 \\
0 & 0 & 0 & 0 & 0 & M_{2}\left(t_{2}, t_{1}\right) \\
0 & 0 & 0 & 0 & M_{2}\left(t_{1}, t_{2}\right) & 0
\end{array}\right) .
$$

The matrices $M_{k}\left(t_{i}, t_{j}\right), k=1,2$ and $1 \leq i \neq j \leq 3$, are given by

$$
M_{1}\left(t_{i}, t_{j}\right)=\left(\begin{array}{ccc}
1-z_{j} & z_{i} & 0 \\
1 & 0 & 0 \\
0 & 0 & 1
\end{array}\right) \quad \text { and } \quad M_{2}\left(t_{i}, t_{j}\right)=\left(\begin{array}{ccc}
1 & 0 & 0 \\
0 & 1-z_{j} & z_{i} \\
0 & 1 & 0
\end{array}\right) \text {. }
$$

Here $z_{i}=\lambda^{t_{i}}$ and $z_{j}=\lambda^{t_{j}}$. In other words, the first column of $\alpha\left(\sigma_{1}\right)$ corresponds to $d\left(\sigma_{1}^{*}\right) p_{\theta}^{t}$, where $t=\left(t_{1}, t_{2}, t_{3}\right)$. We observe that if $X \in T_{p_{\theta}^{t}}(R) \subset V_{t}$ then $d\left(\sigma_{1}^{*}\right)_{p_{\theta}^{t}} \in T_{p_{\theta}^{\sigma_{1}(t)}}(R)=T_{p_{\theta}^{\left(t_{2}, t_{1}, t_{3}\right)}}(R)$. Since the tuples are arranged in the lexicographic order, it follows that the matrix $M_{1}\left(t_{1}, t_{2}\right)$ comes third in the first column. The same is true for all the other square matrices $M_{k}\left(t_{i}, t_{j}\right)$.

\section{Multi-Parameter Representation and Hecke Algebra}

The Hecke algebra is an algebra defined by generators $g_{1}, \ldots, g_{n-1}$ with relations

$$
\begin{gathered}
g_{i} g_{j}=g_{j} g_{i}|i-j| \geq 2, \\
g_{i} g_{i+1} g_{i}=g_{i+1} g_{i} g_{i+1} \quad 1 \leq i \leq n-2, \\
g_{i}^{2}=(q-1) g_{i}+q \quad 1 \leq i \leq n-1 .
\end{gathered}
$$

Here $q$ ia a parameter.

In this section, we show that the multi-parameter representation does not arise from any Hecke algebra.

Lemma 5. The number of eigenvalues of a $k \times k$ matrix $A$ is at least the number of eigenvalues of the matrix $A^{2}$.

Proof. Let $x$ be an eigenvalue of $A^{2}$ then $\operatorname{Det}\left(A^{2}-x I_{k}\right)=0$. Since $A^{2}-x I_{k}=\left(A-\sqrt{x} I_{k}\right)(A+$ $\left.\sqrt{x} I_{k}\right)$, it follows that $\operatorname{Det}\left(\left(A-\sqrt{x} I_{k}\right)\left(A+\sqrt{x} I_{k}\right)\right)=0$ which is equivalent to $\operatorname{Det}\left(\left(A-\sqrt{x} I_{k}\right)\right)=0$ or $\operatorname{Det}\left(\left(A+\sqrt{x} I_{k}\right)\right)=0$. This implies that $\sqrt{x}$ or $-\sqrt{x}$ (or both) are eigenvalues of $A$. 
We now prove a result concerning the pure braid group, which will be used later to show that certain complex specializations of the multi-parameter representation do not arise from any Hecke algebra.

Lemma 6. If there exist integers $k, l, r, s \in\{1, \ldots, n\}$ such that $z_{k} z_{l} \neq z_{r} z_{s}$ and neither one of them equals 1 then $\alpha: P_{n} \rightarrow G L_{m}(C)$ has more than 2 distinct eigenvalues. ( $m=n ! n$ and $n \geq 3$ ).

Proof. For $1 \leq i \neq j \leq n$, we consider $N_{i j}=M_{1}\left(t_{i}, t_{j}\right) M_{1}\left(t_{j}, t_{i}\right), 1 \leq i \neq j \leq n$. The matrix corresponding to $N_{i j}$ is

$$
N_{i j}=\left(\begin{array}{cc}
1-z_{j}+z_{i} z_{j} & z_{j}\left(1-z_{j}\right) \\
1-z_{i} & z_{j}
\end{array}\right) \oplus I_{n-2}
$$

It is easy to see that $A_{12}=\sigma_{1}^{2}$ is direct sum of $N_{i j}$, where each block $N_{i j}$ occurs $(n-2)$ ! times. The characteristic equation of $A_{12}$ is $\operatorname{Det}\left(A_{12}-x I_{m}\right)=0$, which is equivalent to

$$
\prod_{1 \leq i \neq j \leq n} \operatorname{Det}\left(N_{i j}-x I_{n}\right)=\prod_{1 \leq i<j \leq n}\left(\operatorname{Det}\left(N_{i j}-x I_{n}\right)\right)^{2}=0 .
$$

Since $\operatorname{Det}\left(N_{i j}-x I_{n}\right)=(1-x)^{n-1}\left(z_{i} z_{j}-x\right)$, it follows that the characteristic equation of $A_{12}$ is

$$
(1-x)^{n !(n-1)} \prod_{1 \leq i<j \leq n}\left(z_{i} z_{j}-x\right)^{(n-2) ! 2}
$$

We have that 1 and $z_{i} z_{j}$ are eigenvalues of $A_{12}$ for every $1 \leq i \neq j \leq n$. Here, $z_{i} z_{j}$ has multiplicity $(n-2) ! 2$ because $\operatorname{Det}\left(N_{i j}-x I_{n}\right)=\operatorname{Det}\left(N_{j i}-x I_{n}\right)=(1-x)^{n-1}\left(z_{i} z_{j}-x\right)$ and the fact that $N_{i j}$ occurs $(n-2)$ ! times in the matrix corresponding to $A_{12}$.

It is easy to see, by our hypothesis, that $1, z_{k} z_{l}$ and $z_{r} z_{s}$ are distinct eigenvalues of $A_{12}$.

Direct computations show that $\left(N_{i j}-I_{n}\right)\left(N_{i j}-z_{r} z_{s} I_{n}\right)=\left(z_{i} z_{j}-z_{r} z_{s}\right)\left(N_{i j}-I_{n}\right), 1 \leq r<$ $s \leq n$ and $\left(N_{i j}-I_{n}\right)\left(N_{i j}-z_{i} z_{j} I_{n}\right)$ is the $n \times n$ zero matrix. This implies that the minimal polynomial of $A_{12}$ is $(x-1) \prod_{1 \leq i<j \leq n}\left(x-z_{i} z_{j}\right)$.

Theorem 7. If there exist integers $k, l, r, s \in\{1, \cdots, n\}$ such that $z_{k} z_{l} \neq z_{r} z_{s}$ and neither one of them equals 1 then $\alpha: B_{n} \rightarrow G L_{m}(C)$ does not arise from any Hecke algebra. $(m=n ! n$ and $n \geq 2$ ).

Proof. We consider the following cases:

Case $n=2$ : The matrix corresponding to $\sigma_{1}$ is $\alpha\left(\sigma_{1}\right)=\left(\begin{array}{cccc}0 & 0 & 1-z_{1} & z_{2} \\ 0 & 0 & 1 & 0 \\ 1-z_{2} & z_{1} & 0 & 0 \\ 1 & 0 & 0 & 0\end{array}\right)$. 
The minimal polynomial of $\alpha\left(\sigma_{1}\right)$ is $\left(x^{2}-1\right)\left(x^{2}-z_{1} z_{2}\right)$. It is clear that $\alpha\left(\sigma_{1}\right)$ has 4 distinct eigenvalues namely, $-1,1,-\sqrt{z_{1} z_{2}}$ and $\sqrt{z_{1} z_{2}}$.

Case $n>2$ : Using our hypothesis, Lemma 6 asserts that the representation restricted to $P_{n}$ has more than 2 distinct eigenvalues. We then conclude, by Lemma 5, that the representation $\alpha$ defined on the braid group $B_{n}$ has more than 2 distinct eigenvalues.

Since the generators of the Hecke algebra satisfy a quadratic equation, it follows that it has at most 2 distinct eigenvalues and so $\alpha$ does not arise from any Hecke algebra.

\section{Multi-parameter representation is unitary}

Notation 8. Let $(*): M_{m}\left(\mathbb{C}\left[t^{ \pm 1}\right]\right)$ be an involution defined as follows:

$$
\left(f_{i j}(t)\right)^{*}=f_{j i}\left(t^{-1}\right), f_{i j}(t) \in \mathbb{C}\left[t^{ \pm 1}\right]
$$

Definition 9. Let $X$ and $U$ be elements of $G L_{m}(\mathbb{C})$ where $m=n ! n . U$ is called a unitary element (relative to $X$ ) if $U X U^{*}=X$.

Since the matrices corresponding to the generators of $B_{n}$ under the multi-parameter representation are large and of degree $n ! n$, we rather perform our computations only in the case $n=3$. First, we show that the complex specialization of the multi-parameter representation is reducible. Then, we determine sufficient conditions under which the complex specializations of the reducible multi-parameter representation is unitary relative to some invertible hermitian matrix. Since the representation is reducible, it follows that the choice of the hermitian matrix is not unique.

Theorem 10. The multi-parameter representation $\alpha: B_{3} \rightarrow G L_{18}(C)$ is reducible.

Proof. We consider two cases for $z_{i}$.

If $z_{i}=z_{j}$ for all $i, j \in\{1,2,3\}$ then we consider the invariant subspace generated by the column vector $(1, \ldots, 1)^{T}$, where $\mathrm{T}$ is the transpose.

If there exist $i \neq j$ such that $z_{i} \neq z_{j}$ then we consider the invariant subspace generated by the column vector $\left(v_{1}, v_{2}, v_{3}, v_{4}, v_{5}, v_{6}\right)^{T}$, where $v_{1}=\left(z_{1}-1, z_{2}-1, z_{3}-1\right)^{T}, v_{2}=\left(z_{1}-1, z_{3}-1, z_{2}-\right.$ $1)^{T}, v_{3}=\left(z_{2}-1, z_{1}-1, z_{3}-1\right)^{T}, v_{4}=\left(z_{2}-1, z_{3}-1, z_{1}-1\right)^{T}, v_{5}=\left(z_{3}-1, z_{1}-1, z_{2}-1\right)^{T}$ and $v_{6}=\left(z_{3}-1, z_{2}-1, z_{1}-1\right)^{T}$.

Theorem 11. The multi-parameter representation $\alpha: B_{3} \rightarrow G L_{18}(\mathbb{C})$ is unitary under any of the following conditions: 
(i) $z_{1} \neq z_{2}, z_{1}^{2} z_{3} \neq 1$ and $z_{i} \neq 1(i=1,2,3)$.

(ii) $z_{1}=z_{2}=z_{3}$.

(iii) $z_{1}=z_{2}=1$ and $z_{3} \neq 1$.

(iv) $z_{1}=z_{3}=1$ and $z_{2} \neq 1$.

(v) $z_{2}=z_{3}=1$ and $z_{1} \neq 1$.

Proof. (i) $z_{1} \neq z_{2}, z_{1}^{2} z_{3} \neq 1$ and $z_{i} \neq 1(i=1,2,3)$. Let $H=\oplus_{i=1}^{6} H_{i}$, where

$$
\begin{aligned}
& H_{1}=\left(\begin{array}{ccc}
\frac{z_{1}^{2}-z_{2}}{z_{1}\left(-1+z_{2}\right)} & 1 & \frac{z_{2}\left(-1+z_{3}\right)}{z_{3}\left(-1+z_{2}\right)} \\
1 & 1 & \frac{z_{2}\left(-1+z_{3}\right)}{z_{3}\left(-1+z_{1}\right)} \\
\frac{-1+z_{3}}{-1+z_{2}} & \frac{\left(-1+z_{3}\right) z_{1}}{\left(-1+z_{1}\right) z_{2}} & \frac{\left(-1+z_{3}\right)\left(-z_{2}+z_{1} z_{3}\right)}{\left(-1+z_{1}\right)\left(-1+z_{2}\right) z_{3}}
\end{array}\right), H_{2}=\left(\begin{array}{ccc}
\frac{z_{1}^{2}-z_{2}}{z_{1}\left(-1+z_{2}\right)} & \frac{z_{2}\left(-1+z_{3}\right)}{z_{3}\left(-1+z_{2}\right)} & 1 \\
\frac{-1+z_{3}}{-1+z_{2}} & \frac{\left(-1+z_{3}\right)\left(-z_{2}+z_{1} z_{3}\right)}{\left(-1+z_{1}\right)\left(-1+z_{2}\right) z_{3}} & \frac{\left(-1+z_{3}\right)}{\left(-1+z_{1}\right)} \\
1 & \frac{\left(-1+z_{3}\right) z_{1}}{\left(-1+z_{1}\right) z_{3}} & 1
\end{array}\right), \\
& H_{3}=\left(\begin{array}{ccc}
1 & \frac{z_{2}}{z_{1}} & \frac{z_{2}\left(-1+z_{3}\right)}{z_{3}\left(-1+z_{1}\right)} \\
\frac{z_{1}}{z_{2}} & \frac{z_{1}^{2}-z_{2}}{z_{1}\left(-1+z_{2}\right)} & \frac{z_{2}\left(-1+z_{3}\right)}{z_{3}\left(-1+z_{2}\right)} \\
\frac{z_{1}\left(-1+z_{3}\right)}{z_{2}\left(-1+z_{1}\right)} & \frac{\left(-1+z_{3}\right)}{\left(-1+z_{2}\right)} & \frac{\left(-1+z_{3}\right)\left(-z_{2}+z_{1} z_{3}\right)}{\left(-1+z_{1}\right)\left(-1+z_{2}\right) z_{3}}
\end{array}\right), H_{4}=\left(\begin{array}{ccc}
1 & \frac{z_{2}\left(-1+z_{3}\right)}{z_{3}\left(-1+z_{1}\right)} & \frac{z_{2}}{z_{1}} \\
\frac{\left(-1+z_{3}\right) z_{1}}{\left(-1+z_{1}\right) z_{2}} & \frac{\left(-1+z_{3}\right)\left(-z_{2}+z_{1} z_{3}\right)}{\left(-1+z_{1}\right)\left(-1+z_{2}\right) z_{3}} & \frac{z_{2}\left(-1+z_{3}\right)}{z_{1}\left(-1+z_{2}\right)} \\
\frac{z_{1}}{z_{2}} & \frac{\left(-1+z_{3}\right) z_{1}}{\left(-1+z_{2}\right) z_{3}} & \frac{z_{1}^{2}-z_{2}}{z_{1}\left(-1+z_{2}\right)}
\end{array}\right), \\
& \left.\begin{array}{ccc}
\frac{\left(-1+z_{3}\right)\left(-z_{2}+z_{1} z_{3}\right)}{\left(-1+z_{1}\right)\left(-1+z_{2}\right) z_{3}} & \frac{\left(-1+z_{3}\right)}{\left(-1+z_{1}\right)} & \frac{z_{2}\left(-1+z_{3}\right)}{z_{1}\left(-1+z_{2}\right)} \\
\frac{z_{1}\left(-1+z_{3}\right)}{z_{3}\left(-1+z_{1}\right)} & 1 & \frac{z_{2}}{z_{1}} \\
\frac{z_{1}\left(-1+z_{3}\right)}{z_{3}\left(-1+z_{2}\right)} & \frac{z_{1}}{z_{2}} & \frac{z_{1}^{2}-z_{2}}{z_{1}\left(-1+z_{2}\right)}
\end{array}\right) .
\end{aligned}
$$

It is clear that $H_{i}$ is hermitian for every $1 \leq i \leq 6$. We also have that $\operatorname{Det}\left(H_{i}\right)=$ $\frac{\left(-1+z_{3}\right)\left(-1+z_{3} z_{1}^{2}\right)\left(z_{1}-z_{2}\right)^{2}}{\left(-1+z_{1}\right)^{2}\left(-1+z_{2}\right)^{2} z_{3} z_{1}} \neq 0$ by our hypothesis. This implies that $H$ is hermitian and invertible. Also, we have that

$$
\begin{aligned}
\sigma_{1} H \sigma_{1}^{*}= & M_{1}\left(t_{2}, t_{1}\right) H_{3}\left(M_{1}\left(t_{2}, t_{1}\right)\right)^{*} \oplus M_{1}\left(t_{3}, t_{1}\right) H_{5}\left(M_{1}\left(t_{3}, t_{1}\right)\right)^{*} \oplus M_{1}\left(t_{1}, t_{2}\right) H_{1}\left(M_{1}\left(t_{1}, t_{2}\right)\right)^{*} \oplus \\
& M_{1}\left(t_{3}, t_{2}\right) H_{6}\left(M_{1}\left(t_{3}, t_{2}\right)\right)^{*} \oplus M_{1}\left(t_{1}, t_{3}\right) H_{2}\left(M_{1}\left(t_{1}, t_{3}\right)\right)^{*} \oplus M_{1}\left(t_{2}, t_{3}\right) H_{4}\left(M_{1}\left(t_{2}, t_{3}\right)\right)^{*}
\end{aligned}
$$

and

$$
\begin{aligned}
\sigma_{2} H \sigma_{2}^{*}= & M_{2}\left(t_{3}, t_{2}\right) H_{2}\left(M_{2}\left(t_{3}, t_{2}\right)\right)^{*} \oplus M_{2}\left(t_{2}, t_{3}\right) H_{1}\left(M_{2}\left(t_{2}, t_{3}\right)\right)^{*} \oplus M_{2}\left(t_{3}, t_{1}\right) H_{4}\left(M_{2}\left(t_{3}, t_{1}\right)\right)^{*} \oplus \\
& M_{2}\left(t_{1}, t_{3}\right) H_{3}\left(M_{2}\left(t_{1}, t_{3}\right)\right)^{*} \oplus M_{2}\left(t_{2}, t_{1}\right) H_{6}\left(M_{2}\left(t_{2}, t_{1}\right)\right)^{*} \oplus M_{2}\left(t_{1}, t_{2}\right) H_{5}\left(M_{2}\left(t_{1}, t_{2}\right)\right)^{*} .
\end{aligned}
$$

Direct computations show that $\sigma_{1} H \sigma_{1}^{*}=\sigma_{2} H \sigma_{2}^{*}=H$.

(ii) $z_{1}=z_{2}=z_{3}=z$. We consider three cases, $z=1, z=-1$ and $z \neq \pm 1$. 
Case $z=1: \sigma_{1} \sigma_{1}^{*}=\sigma_{2} \sigma_{2}^{*}=I_{18}$.

Case $z=-1: M_{1}\left(t_{r}, t_{s}\right)=\left(\begin{array}{ccc}2 & -1 & 0 \\ 1 & 0 & 0 \\ 0 & 0 & 1\end{array}\right)$ and $M_{2}\left(t_{r}, t_{s}\right)=\left(\begin{array}{ccc}1 & 0 & 0 \\ 0 & 2 & -1 \\ 0 & 1 & 0\end{array}\right)$, where $1 \leq r \neq s \leq 3$.

Let $H=\oplus_{1}^{6} M$ where $M=\left(\begin{array}{ccc}1 & 1+i & 1+i \\ 1-i & 1 & 1+i \\ 1-i & 1-i & 1\end{array}\right)$. Here $i$ is a complex number such that $i^{2}=-1$. Since $M_{1}\left(t_{r}, t_{s}\right) M\left(\left(M_{1}\left(t_{r}, t_{s}\right)\right)^{*}=M_{2}\left(t_{r}, t_{s}\right) M\left(\left(M_{2}\left(t_{r}, t_{s}\right)\right)^{*}=M\right.\right.$, it follows that $\sigma_{1} H \sigma_{1}^{*}=$ $\sigma_{2} H \sigma_{2}^{*}=H$. Note that $H$ is hermitian and invertible whose determinant is equal to one.

Case $z \neq \pm 1 . M_{1}\left(t_{r}, t_{s}\right)=\left(\begin{array}{ccc}1-z & z & 0 \\ 1 & 0 & 0 \\ 0 & 0 & 1\end{array}\right)$ and $M_{2}\left(t_{r}, t_{s}\right)=\left(\begin{array}{ccc}1 & 0 & 0 \\ 0 & 1-z & z \\ 0 & 1 & 0\end{array}\right)$, where $1 \leq r \neq s \leq 3$.

Let $H=\oplus_{1}^{6} M$, where $M=\left(\begin{array}{ccc}0 & 1+z & 1+z \\ \frac{1+z}{z} & 0 & 1+z \\ \frac{1+z}{z} & \frac{1+z}{z} & 0\end{array}\right)$. It is easy to see that $M_{1}\left(t_{r}, t_{s}\right) M\left(M_{1}\left(t_{r}, t_{s}\right)\right)^{*}=M$ and $M_{2}\left(t_{r}, t_{s}\right) M\left(M_{2}\left(t_{r}, t_{s}\right)\right)^{*}=M$. Therefore, we get that $\sigma_{1} H \sigma_{1}^{*}=\sigma_{2} H \sigma_{2}^{*}=H$, where $H$ is an invertible hermitian matrix whose determinant is equal to $\frac{(z+1)^{24}}{z^{12}}$. It is worth to mention that $M_{k}\left(t_{i}, t_{j}\right)$ is the Burau representation $(k=1,2$ and $1 \leq i \neq j \leq 3)$.

(iii) $z_{1}=z_{2}=1$ and $z_{3} \neq 1$. Let $H=\oplus_{i=1}^{6} H_{i}$, where

$$
H_{3}=H_{1}=\left(\begin{array}{ccc}
1 & 0 & -1 \\
0 & 1 & -1 \\
-1 & -1 & 1
\end{array}\right), H_{4}=H_{2}=\left(\begin{array}{ccc}
1 & -1 & 0 \\
-1 & 1 & -z_{3} \\
0 & \frac{-1}{z_{3}} & 1
\end{array}\right) \text { and } H_{6}=H_{5}=\left(\begin{array}{ccc}
1 & -z_{3} & -z_{3} \\
\frac{-1}{z_{3}} & 1 & 0 \\
\frac{-1}{z_{3}} & 0 & 1
\end{array}\right) .
$$

It is easy to see that $\sigma_{1} H \sigma_{1}^{*}=\sigma_{2} H \sigma_{2}^{*}=H$, where $H$ is an invertible hermitian matrix whose determinant is equal to one.

(iv) $z_{1}=z_{3}=1$ and $z_{2} \neq 1$. Let $H=\oplus_{i=1}^{6} H_{i}$, where

$$
H_{6}=H_{1}=\left(\begin{array}{ccc}
1 & -1 & 0 \\
-1 & 1 & -z_{2} \\
0 & \frac{-1}{z_{2}} & 1
\end{array}\right), H_{5}=H_{2}=\left(\begin{array}{ccc}
1 & 0 & -1 \\
0 & 1 & -1 \\
-1 & -1 & 1
\end{array}\right) \text { and } H_{4}=H_{3}=\left(\begin{array}{ccc}
1 & -z_{2} & -z_{2} \\
\frac{-1}{z_{2}} & 1 & 0 \\
\frac{-1}{z_{2}} & 0 & 1
\end{array}\right) \text {. }
$$

It is easy to see that $\sigma_{1} H \sigma_{1}^{*}=\sigma_{2} H \sigma_{2}^{*}=H$, where $H$ is an invertible hermitian matrix whose determinant is equal to one. 
(v) $z_{2}=z_{3}=1$ and $z_{1} \neq 1$. Let $H=\oplus_{i=1}^{6} H_{i}$, where

$$
H_{2}=H_{1}=\left(\begin{array}{ccc}
1 & z_{1} & z_{1} \\
\frac{1}{z_{1}} & -1 & 0 \\
\frac{1}{z_{1}} & 0 & -1
\end{array}\right), H_{5}=H_{3}=\left(\begin{array}{ccc}
-1 & 1 & 0 \\
1 & 1 & z_{1} \\
0 & \frac{1}{z_{1}} & -1
\end{array}\right) \text { and } H_{4}=H_{6}=\left(\begin{array}{ccc}
-1 & 0 & 1 \\
0 & -1 & 1 \\
1 & 1 & 1
\end{array}\right) .
$$

It is easy to see that $\sigma_{1} H \sigma_{1}^{*}=\sigma_{2} H \sigma_{2}^{*}=H$, where $H$ is an invertible hermitian matrix whose determinant is equal to $3^{6}$.

\section{References}

[1] S. Bigelow and J. P. Tian, Generalized Long- Moody representations of braid groups, Communications in Contemporary Math, 10(2008), (suppl.1), 1093-1102.

[2] J. S. Birman, Braids, Links and Mapping Class Groups. Annals of Mathematical Studies. Princeton University Press, 82, New Jersey, 1975.

[3] D.D. Long, On the linear representation of braid groups. Transaction of the American Mathematical Society, 311(1989), 535-560

[4] V. Shpilrain, Representing Braids by Automorphisms, International Journal of Algebra and Computation, 11 (2001), 773-777.

[5] C. C. Squier, The Burau representation is unitary, Proceedings of the American Mathematical Society, 90(1984), 199-202.

[6] J. P. Tian, Spin representations of Artin's braid group, Preprint, 2007.

Department of Mathematics, Beirut Arab University, P.O. Box 11-5020, Beirut, Lebanon.

E-mail: maa531@bau.edu.lb

Department of Mathematics, Beirut Arab University, P.O. Box 11-5020, Beirut, Lebanon.

E-mail:mna@bau.edu.lb

Department of Computer Science and Mathematics, Lebanese American University, P.O. Box 13-5053 Chouran, Beirut, Lebanon.

E-mail: shabre@lau.edu.lb 